\title{
Quantization noise and its reduction in lensless Fourier digital holography
}

\author{
Nitesh Pandey* and Bryan Hennelly \\ Department of Computer Science, National University of Ireland, Maynooth, Kildare, Ireland \\ ${ }^{*}$ Corresponding author: npandey@cs.nuim.ie
}

Received 30 August 2010; accepted 3 November 2010;

posted 11 November 2010 (Doc. ID 134120); published 10 January 2011

\begin{abstract}
Digital holography is an imaging technique that enables recovery of topographic 3D information about an object under investigation. In digital holography, an interference pattern is recorded on a digital camera. Therefore, quantization of the recorded hologram is an integral part of the imaging process. We study the influence of quantization error in the recorded holograms on the fidelity of both the intensity and phase of the reconstructed image. We limit our analysis to the case of lensless Fourier off-axis digital holograms. We derive a theoretical model to predict the effect of quantization noise and we validate this model using experimental results. Based on this, we also show how the resultant noise in the reconstructed image, as well as the speckle that is inherent in digital holography, can be conveniently suppressed by standard speckle reduction techniques. We show that high-quality images can be obtained from binary holograms when speckle reduction is performed. (C) 2011 Optical Society of America
\end{abstract}

OCIS codes: $\quad 090.1995,110.2970,110.4280,070.0070$.

\section{Introduction}

The idea of recording and reconstructing holograms digitally was proposed in early studies by Goodman and Lawrence [1], Konrod et al. [2], and Yaroslavskii [3]. In later years, it was further developed by Schnars and Juptner [4]. In digital holography, a coherent reference wave is incident on a twodimensional (2D) image sensor, where it forms an interference pattern with coherent light reflected or transmitted through an object of interest. The resulting interference pattern, called the digital hologram (DH), contains encoded information about the threedimensional (3D) topographic features of the object. The DH can be transmitted remotely to another place and the 3D object can be reconstructed and displayed remotely. This information can be analyzed with numerous digital processing methods. The advantage of numerical processing has helped digital holography become a fast growing field with numerous applications including aberration-compensated phase-contrast microscopy $[\underline{5}, \underline{6}]$, shape measurement

0003-6935/11/070B58-13\$15.00/0

(ㄷ) 2011 Optical Society of America
[7], and 3D display [8]. Recently, the resolution of $\overline{\mathrm{CCD}}$ /CMOS sensors has increased significantly and with it, so, too, has the computational requirement of a digital holographic imaging system. The bandwidth needed for transmission of holograms from one point to another has also increased, and hence compression of digital holograms has been an active area of research [9-18]. In this context, the quantization of the hologram and the effect on the reconstructed images has been studied before.

Reducing the number of quantization levels to represent the hologram has various advantages. It directly reduces the memory requirement of the hologram, and combined with suitable processing and hardware, can decrease the computational requirement of the digital imaging system [9]. A quantized hologram also can be compressed effectively. However, as a side effect, the quantization introduces a quantization noise in the hologram and the reconstructed image. This can have an adverse effect on the quality of the reconstructed intensity for 3D display applications, and on the fidelity of the phase information, in the case of digital holographic microscopy. The advantages and disadvantages of quantization in digitally recorded holograms in various 
recording architectures have been discussed by a number of authors. Naughton et al. [10] have demonstrated the effectiveness of quantization in combination with lossy and lossless compression techniques for transmission and storage of digital holograms. A networked "real time Fresnel field transmission system" was developed by the authors in [11] to test different compression algorithms. They obtained the complex wavefield by phase shifting, and then quantized and compressed the real and imaginary parts separately. In [12], quantization of the real and imaginary parts of the complex hologram values (obtained from phase shifting) was studied in combination with lossless and lossy compression techniques. In [13], reconstruction from pure phase objects was studied, and perceptible reconstructions were achieved by using a 1 bit (binary) representation level. Various other quantization schemes were developed and studied by Shortt et al. [14-16]. An in-depth simulation and experimental study on the effect of quantization in phase-shifting digital holography was performed by Mills and Yamaguchi [17], in which the individual phase-shifted interferograms were quantized before calculation of the complex wavefield. They found the use of 4 bits for hologram quantization to be adequate for "visual recognition" of the reconstructed images. They also mentioned that using 2 bits per pixel (4 levels) gives noisy reconstructions. In phase-shifting digital holography, Darakis et al. studied the effect of compression in the individual interferograms, the complex hologram, and the reconstruction [18,19]. Also see [20] for a survey on quantization in holography.

The lowest quantization one can achieve directly is 1 bit per pixel, the binary hologram. This rate introduces appreciable noise in the reconstructed image. If the noise in the reconstructed image can be reduced, there are several advantages of using binary holograms for display and projection applications. Ferroelectric binary spatial light modulators (SLMs) have very fast switching rates (up to $10000 \mathrm{fps}$ ) and can display holograms at a fast frame rate. Binary holograms occupy considerably less memory (90\% less than an 8 or 12 bit per pixel hologram). Also, in principle, it is faster to acquire binary holograms. Such advantages of optical setups employing binary SLMs have been shown in $[21,22]$. Another imaging strategy employing binary sensors and compressive sensing was recently shown in [23].

In the 1960-70s, various authors studied the influence of quantization arising from the finite printer resolution in computer generated holograms and their efficiency [24-26]. In [27], Powers and Goodman derive the probability of error in holographic memory employing computer-generated holograms when there is a quantization error in the hologram plane. Binary holograms were carefully studied [28] because of the ease of printing, and new methods were developed for synthesis of binary CGHs [29].
The main contributions of this paper are:

- We analyze quantization noise in lensless Fourier digital holographic imaging. In this architecture, the reconstruction is obtained by a single discrete Fourier transform (DFT) of the recorded hologram. We believe that the results can be extended to the Fresnel case. To the best of our knowledge, this is the first time that quantization has been studied for the lensless digital holography architecture. Furthermore, our system is off-axis. Off-axis is preferred over phase-shifting techniques for imaging dynamic events, as it involves a single capture.

- We derive a simple theoretical model that predicts the effect of quantizing the digital hologram on both the intensity and phase values of the reconstructed image.

- We confirm the theoretical model, using experimental results that closely agree. In those instances where they do not agree, we observe, for the first time in digital holography, the phenomenon of banding that occurs in highly quantized signals [30].

- Following from our theoretical model, we propose and experimentally validate a method to reduce the noise in the reconstructed intensity resulting from quantization, using a speckle-reduction technique. This method has the added advantage of also reducing speckle inherent in holographic imaging.

- Additionally, we briefly mention the other noise sources that can occur in DH imaging, such as channel noise, shot noise, thermal noise, and computational noise, and we discuss methods for reducing their influence using what we refer to as "speckle reduction-like approaches."

In Section 2, we discuss the basics of off-axis lensless Fourier digital holography and our experimental setup. In Section 3 we develop our theoretical model and compare it with experimental results. Finally, in Section $\underline{4}$, we reduce this error and the inherent speckle noise by adding multiple reconstructions and quantifying the reduction, and give a brief conclusion.

\section{Lensless Digital Fourier Holography}

\section{A. Theory}

Lensless Fourier digital holography involves the interference of the object wavefield with a spherical reference wave originating from a point source located at the same distance away from the sensor as the object. A lensless Fourier digital hologram can be represented by a sum of four interference terms. If $O$ and $R$ represent the reference wave field and the complex amplitude of the object wave at the hologram plane respectively, then the hologram, $H$ can be represented by

$$
H(x)=|O(x)|^{2}+|R(x)|^{2}+O(x) R(x)^{*}+R(x) O(x)^{*}
$$


For simplicity, we use a one-dimensional (1D) representation. The hologram, $H$, contains the object information, $O$, in the third term. When the reconstruction operation is performed on $H$, the other three terms contribute to the zero-order noise and the twin image. Here we briefly describe the reconstruction process in Fourier digital holography. For a thorough evaluation, we refer the reader to [31].

The relationship between the wavefield at the hologram plane, $O$, and the object plane, $o$, is given by the Fresnel transform under the paraxial approximation:

$$
O(x)=\exp \left(\frac{j \pi x^{2}}{\lambda d}\right) F\left\{o\left(x^{\prime}\right)\left(\exp \frac{j \pi x^{\prime 2}}{\lambda d}\right)\right\}(x) .
$$

The reference wave from a point source displaced laterally by $\xi$ and placed at a distance $d$ away is represented by $R=\exp \left(\frac{i \pi(x-\xi)^{2}}{\lambda d}\right)$. The reconstruction can be written as

$$
\begin{aligned}
F\{H(x)\}\left(x^{\prime}\right)= & F\left\{|O(x)|^{2}\right\}\left(x^{\prime}\right)+\delta\left(x^{\prime}\right) \\
& +o^{*}\left(\xi-x^{\prime}\right) \exp \left(\frac{j \pi\left(\xi-x^{\prime}\right)^{2}}{\lambda d}\right) \\
& +o\left(x^{\prime}-\xi\right) \exp \left(\frac{-j \pi\left(x^{\prime}-\xi\right)^{2}}{\lambda d}\right) .
\end{aligned}
$$

The Fourier transform of the reference beam is a Dirac delta function, $\delta\left(x^{\prime}\right)$. As long as $o\left(x^{\prime}\right)$ has a finite extent and $\xi$ is sufficiently large, we can expect the latter two terms to be spatially separated from the first two terms, which are known as the zeroorder terms and which occupy an area in the center of our reconstruction. In this way, the complex object wavefield $o\left(x^{\prime}\right)$ can be recovered. The reconstructed complex image, aside from a quadratic phase factor, is thus given by the inverse Fourier transform of the hologram. This is achieved by calculating the discrete Fourier transform (DFT) using the FFT algorithm. A comprehensive analysis of the extent of these four terms in Eq. (3) can be found in [31].

When a digital hologram is acquired with a discrete CCD/CMOS sensor and reconstructed digitally, at least four physical effects impact on the image formation and image quality in the reconstruction plane. They are:

1. The camera aperture size, which determines the point-spread function of the imaging system.

2 . The averaging effect on the individual pixels due to their finite area.

a. Averaging in the final complex image, in the case of a Fresnel holographic imaging system.

b. A sinc amplitude modulation in the reconstruction plane, in the case of a Fourier or lensless Fourier imaging system.

3 . The sampling rate of the $2 \mathrm{D}$ image sensor, which results in repeating copies of the image in the reconstruction plane.
4. The quantization of the pixel values, i.e., the pixel values are quantized in accordance with the bit-depth of the memory registers in the detection system. The bit-depth of the camera is typically $8-12$ bits per pixel.

The effects of the first three physical effects on the reconstructed image have been well studied and analyzed $[32,33]$. The fourth effect is studied here.

\section{B. Experimental Setup}

A schematic for the experimental setup for lensless Fourier digital holography is shown in Fig. 1. We use a Mach-Zehnder interferometer employing a diode-pumped solid state laser $(\lambda=785 \mathrm{~nm})$ to record off-axis lensless Fourier digital holograms. The spherical reference beam is generated by placing a lens (focal length of $10 \mathrm{~cm}$ ) in the path of a plane-collimated reference beam such that the lens generates a point source at the same distance as the object is from the camera. We use a 12 bit camera (AVT Firewire) with pixel pitch $6.45 \mu \mathrm{m}$ and size $1040 \times$ 1392 pixels for recording. Using this experimental setup, we record a lensless Fourier hologram of a 2D object, a USAF resolution chart. Since we are studying quantization, we intentionally use the full dynamic range offered by the camera by adjusting the power in the beams. This results in some saturated pixels. The percentage of saturated pixels is $0.5 \%$, and thus has little effect on the reconstructed image. This hologram is quantized at 12 bit precision by the camera and is our benchmark hologram for comparing the effect of further quantization. Figure 2(a) shows the original 12 bit hologram; Fig. 2(b), the histogram of the pixel values in the hologram; Fig. 2(c), the reconstructed image; and Fig. $2(\mathrm{~d})$, a section of the reconstructed image.

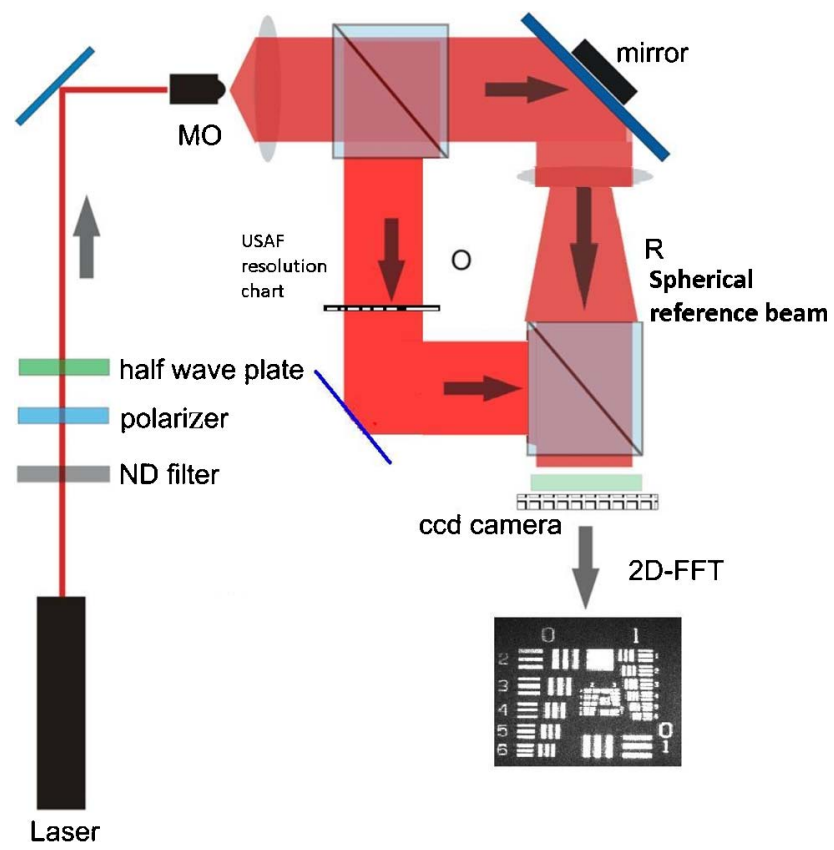

Fig. 1. (Color online) Setup for Fourier digital holography. 


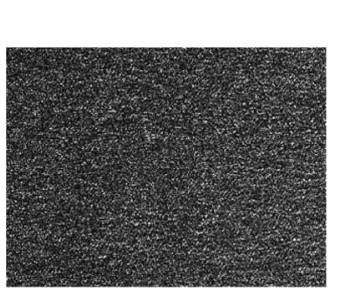

Hologram

(a)

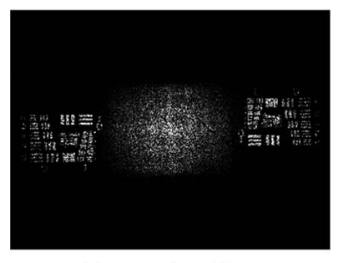

Reconstruction

(c)

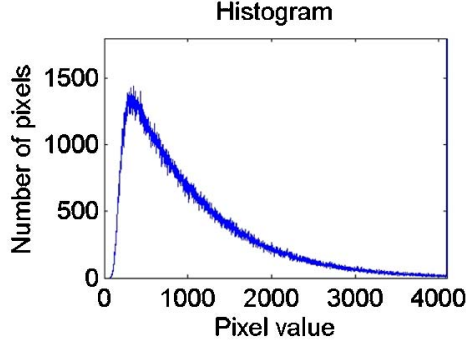

(b)

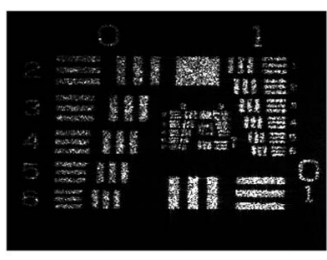

Object area

(d)

Fig. 2. (Color online) (a) Original hologram. (b) Histogram showing the grayscale value and the corresponding number of pixels. (c), (d) Reconstructed image.

\section{A Model for Quantization Noise in Fourier Digital Holography}

\section{A. Noise Due to Quantization in the Hologram Plane}

The image acquired by any digital image sensor is in a quantized format. It can be further quantized computationally to suit the needs of the application, e.g compression. In this paper, we base our experimental results on digital holograms that are obtained by digitally requantizing our benchmark 12 bit hologram to a reduced number of quantization levels. We use the following notation, which is illustrated in Fig. 3 , throughout this paper.

- $H$ represents the ideal $2 \mathrm{D}$ hologram and $h$ represents its ideal reconstruction. $H_{l}$ represents the requantized $2 \mathrm{D}$ hologram. $h_{l}$ represents its reconstruction. $l$ denotes the number of bits used to quantize the hologram.

- $Q_{l}$ represents the noise in the hologram plane due to quantization with $l$ bits. It is a $2 \mathrm{D}$ matrix representing the quantization error per pixel. $q_{l}$ repre-

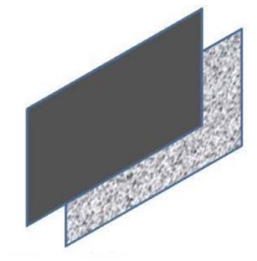

$H+Q$

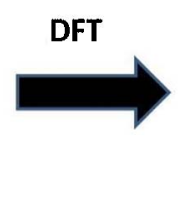

Fig. 3. the hologram plane and the reconstruction (Fourier) plane. $Q_{l}$ stands for the quantization noise in the hologram plane and $q_{l}$ stands for the quantization noise in the Fourier domain. $q_{l}$ is complex and the reconstruction is the complex sum of the ideal reconstruction $h$ and the noise $q_{l}$. sents the resulting quantization noise in the reconstruction plane. $q_{l}$ is the DFT of $Q_{l}$.

When an optical wavefield with a particular variation in irradiance (of dark to bright) is incident onto the image sensor, the image sensor captures this variation in accordance with the quantization rate. During the digital capture process, the camera pixels convert the charge acquired into a voltage, and this voltage is digitized and quantized into a pixel value that is directly indicative of the intensity of light incident on that pixel. The interval between $M_{x}$ and $M_{n}$ is uniformly divided into $2^{l}$ levels and all the pixels are "binned" into their closest level. The quantizing interval thus is

$$
\Delta_{l}=\frac{M_{x}-M_{n}}{2^{l}},
$$

and the quantized hologram thus has $2^{l}$ levels. Quantization results in some loss of information and causes noise in the reconstructed image in both the phase and amplitude. This is depicted in Fig. 4, which shows the degrading effect of uniform quantization on the reconstructed image quality. In Fig. 4(a), we show the histogram of $H_{12}$, the hologram recorded using the full 12 bit range of the camera. In Fig. 4(b), we show the intensity of the resulting numerical reconstruction. In Fig. 4(c), we show the histogram of $H_{8}$, the case where the 12 bit hologram is requantized to 8 bit; in this case, there are 16 times fewer levels than in the 12 bit case. Thus, we see that the number of pixels in each bin increases. In Fig. 4(d), the intensity of the resulting numerical reconstruction is shown. There is no apparent reduction in quality compared with the 12 bit case. In Figs. 4(e) and 4(f), the holograms and the reconstructed image are shown for the 2 bit case where there are just 4 levels. Although the noise has increased, the object is still perceptible. Finally, Figs. $4(\mathrm{~g})$ and $4(\mathrm{~h})$ show the limiting case for quantization, the binary case where the object is immersed in a high amount of quantization noise.

Quantizing in the way described above means that the value of the quantized pixel will deviate from its correct value by $\pm \Delta_{l} / 2$. Thus, we can model the quantization noise, $Q_{l}$, by assuming that it is an additive white noise sequence with values uniformly distributed between $-\Delta_{l} / 2$ and $+\Delta_{l} / 2$, where $\Delta_{l}$ is the quantization interval [34]. In the hologram plane, the quantized hologram can be written as

$$
H_{l}=H+Q_{l}
$$

For such a uniform white noise sequence, the mean value is 0 , as the noise is fluctuating randomly between $-\Delta / 2$ and $+\Delta / 2$, and the variance can be derived as 


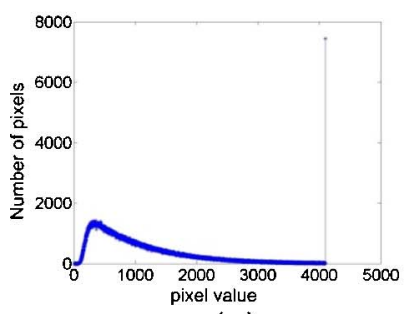

(a)

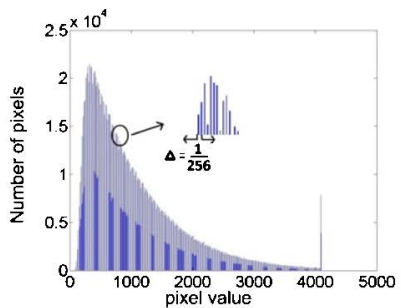

(c)

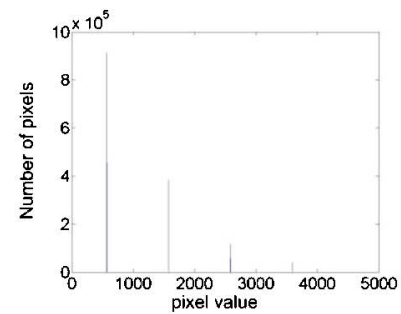

(e)

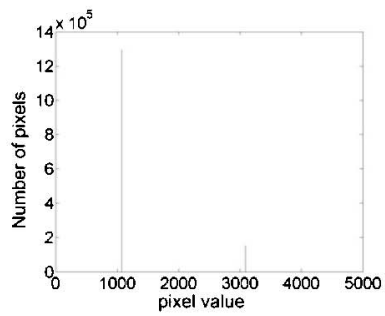

(g)

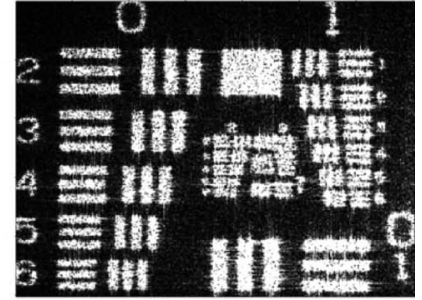

(b)

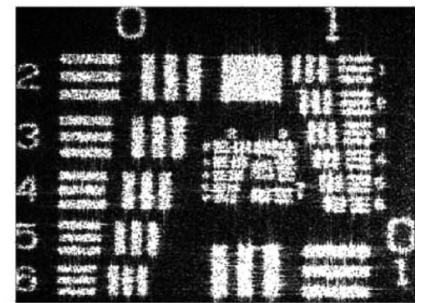

(d)

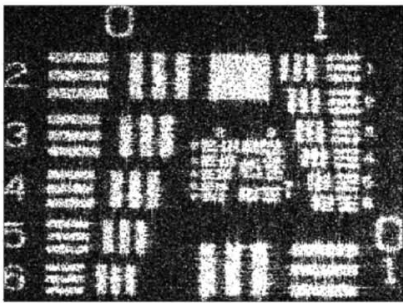

(f)

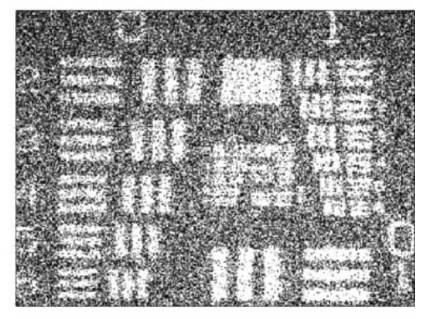

(h)
Fig. 4. (Color online) Histograms for various quantization levels and the corresponding reconstructed images. (a) and (b) 12 bits, (c) and (d) 8 bits, (e) and (f) 4 bits, (g) and (h) 1 bit (binary) cases.

$$
\sigma_{Q_{l}}^{2}=\int_{-\Delta_{l} / 2}^{\Delta_{l} / 2} x^{2}\left(\frac{1}{\Delta_{l}}\right) \mathrm{d} x=\frac{\Delta_{l}^{2}}{12} .
$$

Thus, for a quantizer with interval $\Delta$, the standard deviation in the error is

$$
\sigma_{Q_{l}}=\frac{\Delta_{l}}{\sqrt{12}}=\frac{M_{x}-M_{n}}{2^{l} \sqrt{12}} .
$$

We calculate the quantization error $Q_{l}$ for different values of $l$ in the following way. The acquired $12 \mathrm{bit}$ hologram, $H_{12}$, is requantized down to $l$ bits to generate the lower quantized hologram, $H_{l} . Q_{l}$ is signal independent and additive and $H_{l}$ can be written as $H_{l}=H+Q_{l}$. Since $H_{12} \approx H$, this gives $Q_{l}=H_{l}-H_{12}$. We can thus calculate the quantization noise for various values of $l$ by computing $H_{l}$ and subtracting

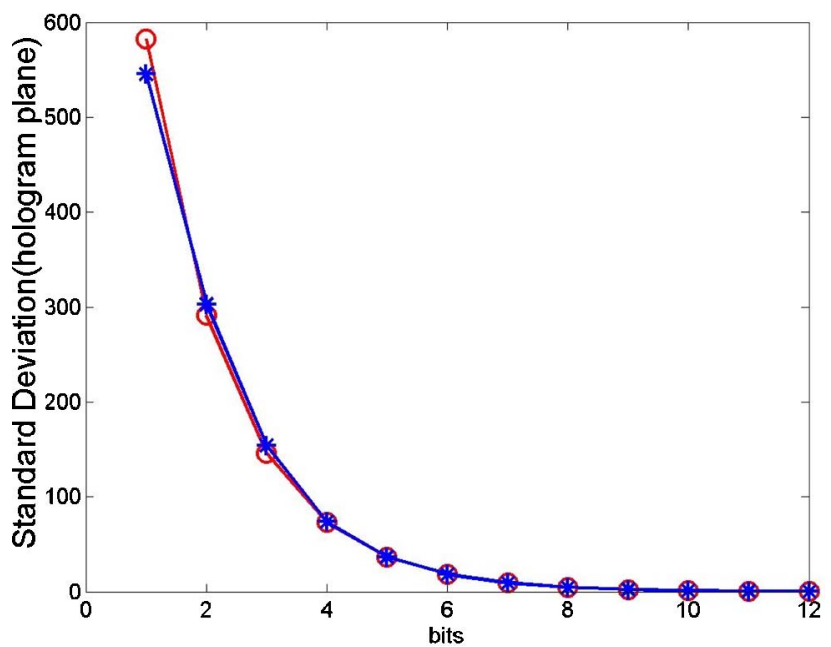

Fig. 5. (Color online) Standard deviation of the quantization noise in the hologram plane versus the theoretical prediction.

it from $H_{12}$. In Fig. 5, the theoretical and actual standard deviation in the quantization noise, $Q_{l}$, for different values of $l$ (from 12 bits to 1 bit) is shown. The standard deviation closely follows Eq. (7). The curves closely match except for the 1 bit case where there is a small deviation from the predicted value. This led us to discover that at very low bit levels, the quantization noise carries with it the same structure as that of the hologram, $H$. This concept is illustrated in Fig. 6, where a simple sinusoidal signal is quantized to 32 levels (5 bits), four levels ( 2 bits) and two levels (1 bit). The quantized signal and the quantization noise are shown for the three cases and it can be seen that when the signal is quantized to the binary case, the quantization noise carries the same

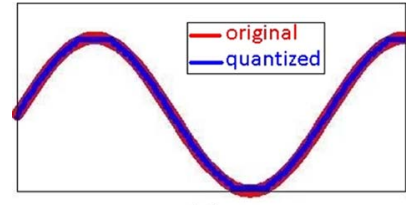

(a)

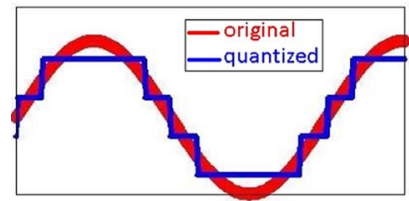

(c)

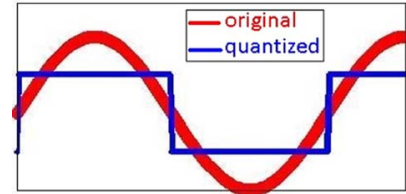

(e)

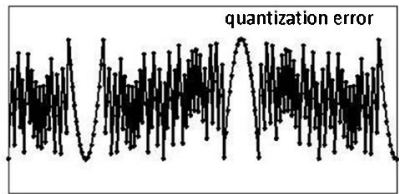

(b)

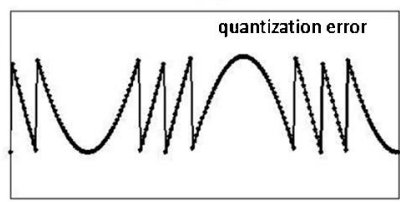

(d)

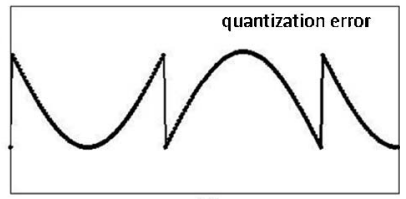

(f)
Fig. 6. (Color online) Quantization of a sinusoidal signal and the corresponding quantization noise for three different cases. (a) and (b) 5 bits (b) and (c) 2 bits and (d) and (e) 1 bit. It can be seen that for 5 bits the quantization noise is almost randomly varying as in (b), and for the 1 bit case in (e), it has the particular characteristic frequency of the original signal. 
characteristic frequency and the shape as that of the signal. Thus the noise is no longer a random white noise but has the characteristic frequency of the signal. This is analogous to the phenomenon of color banding in computer graphics which occurs when there are very few bits per pixel available for representing color. The concept of dithering in signal processing is related to reducing such effects in highly quantized signals [30]. In Fig. 7, we plot the histograms of the quantization noise, $Q_{l}$ for $l$ values of (a) 8 bits, (b) 6 bits (c) 4 bits and (d) 1 bit. The histogram for the 1 bit case is not uniformly distributed and thus $Q_{l}$ will contain many of the same frequency components of $H$ for low values of $l$. This is again clearly demonstrated when we calculate the DFT of $Q_{l}$ for $l$ values of $8,6,4$, and 1 bit. As we have discussed above, the result of quantizing $H$ at a very low quantizing rate results in an error signal, $Q_{l}$, that inherits frequencies of $H$. The DFT of $H$ is the reconstructed image and therefore we can expect the DFT of $Q_{l}$, which we denote as $q_{l}$ to have resemble the reconstructed image. In Fig. $\underline{8}$, it can be seen that for the low value of $l=1$ bit, the object can be seen in the reconstruction of the noise. The "nonwhite noise" occurs only at low quantization rates (around 2 bits and less). For other cases, the assumption that the quantization noise in the hologram plane is a white noise sequence holds strongly and we proceed with this assumption for the rest of our analysis. We refer the reader to [35] for a discussion on the conditions for quantization noise to be uniform and white.

\section{B. Noise Due to Quantization in the Reconstruction Plane}

In the previous subsection it was shown that the noise in the hologram plane can be described as a uniformly distributed white noise with a standard deviation of $\Delta_{l} / \sqrt{12}$. We are interested in studying the properties of $q_{l}$, the resulting noise in the reconstructed image. In lensless Fourier digital holography, the reconstruction is obtained by applying a

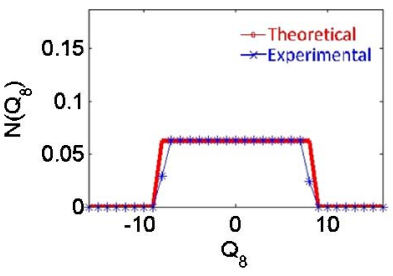

(a)

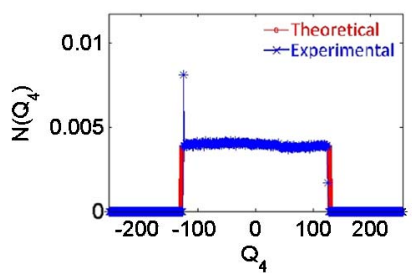

(c)

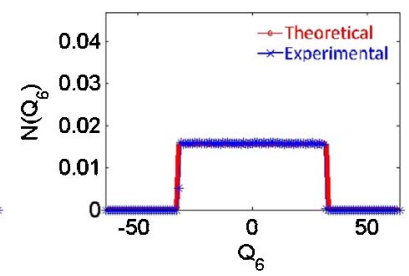

(b)

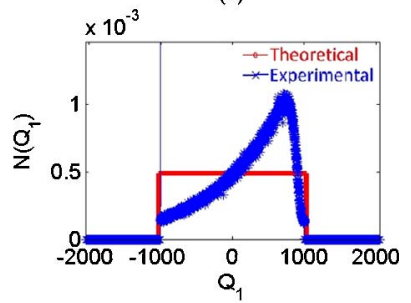

(d)
Fig. 7. (Color online) Normalized histograms of the quantization noise in the hologram plane. (a) 8 bits. (b) 6 bits. (c) 4 bits. (d) 1 bit.

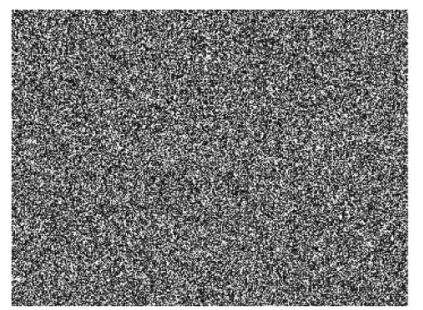

$\mathrm{q}_{8}$

(a)

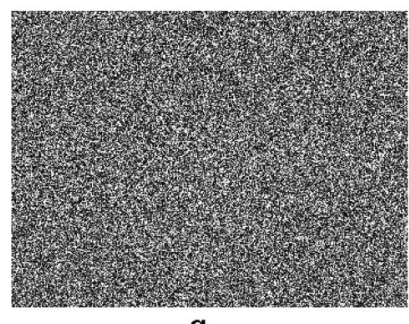

$\mathrm{q}_{4}$

(c)

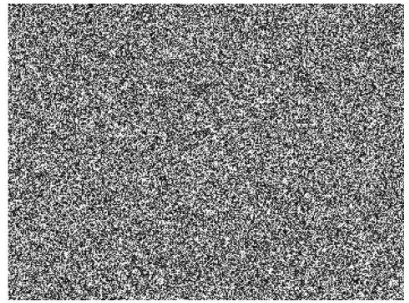

$\mathrm{q}_{6}$

(b)

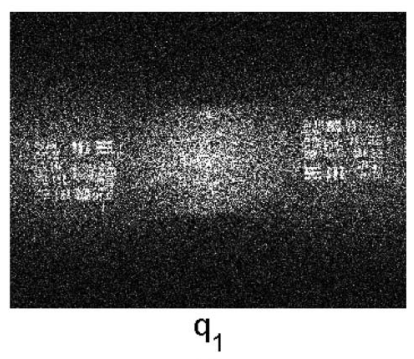

(d)
Fig. 8. For low bit quantization like 1 bit, the quantization noise in the hologram plane is not uniform and white. This can be seen in the reconstruction from the quantization noise for four different cases. (a) 8 bits, (b) 6 bits, (c) 4 bits, (d) 1 bit. The reconstruction of the noise for 1 bit is not random and the object can be seen in it.

DFT to the acquired digital hologram. The discrete Fourier transform is a linear operation, therefore, the noise in the reconstruction plane is the Fourier transform of the noise in the hologram plane. We now proceed to show that the noise in the reconstruction plane shares the same first-order statistical properties as those of speckle. In the case of coherent light incident on a rough surface, the resulting speckle pattern can be treated as a random walk and the first-order statistical properties can be derived under the assumptions that the amplitudes and phases of the random walk components at each point are statistically independent from phasors at other points and also that the amplitudes and phases are independent of each other. A further assumption is that the phases are uniformly distributed between $-\pi$ and $\pi$. In the case of lensless Fourier digital holography, quantization noise in the Fourier plane is the DFT of the noise in the hologram plane. A DFT of white noise can also be considered as a random walk arising from the phases at which the DFT is calculated which are uniformly distributed between $-\pi$ and $\pi$. Thus in the Fourier holographic system, the noise in the reconstruction plane has the same first order statistical properties as that of speckle. We proceed to explicitly derive the statistical properties of this noise:

$$
h_{l}=\operatorname{DFT}\left(H_{l}\right)=\operatorname{DFT}\left(H+Q_{l}\right)=h+q_{l} .
$$

The statistical properties of the DFT of a discrete random signal can be calculated. We derive here the mean and variance of $q_{l}$ which is a sequence of 
complex numbers with the real and imaginary parts given by

$$
\begin{gathered}
q_{l}(k)=R_{l}(k)+i I_{l}(k), \\
R_{l}(k)=\frac{1}{\sqrt{N}} \sum_{n=0}^{N-1} Q_{l}(n) \cos \left(\frac{2 \pi k n}{N}\right), \\
I_{l}(k)=\frac{1}{\sqrt{N}} \sum_{n=0}^{N-1} Q_{l}(n) \sin \left(\frac{2 \pi k n}{N}\right) .
\end{gathered}
$$

The mean of the real and imaginary parts of the noise, $E\left[R_{l}\right]$ and $E\left[I_{l}\right]$, can be found to be

$$
\begin{aligned}
E\left[R_{l}\right] & =\frac{1}{\sqrt{N}} \sum_{n=0}^{N-1} E\left[Q_{l}(n) \cos \left(\frac{2 \pi k n}{N}\right)\right] \\
& =\frac{1}{\sqrt{N}} \sum_{n=0}^{N-1} E\left[Q_{l}(n)\right] E\left[\cos \left(\frac{2 \pi k n}{N}\right)\right]=0, \\
E\left[I_{l}\right] & =\frac{1}{\sqrt{N}} \sum_{n=0}^{N-1} E\left[Q_{l}(n) \sin \left(\frac{2 \pi k n}{N}\right)\right] \\
& =\frac{1}{\sqrt{N}} \sum_{n=0}^{N-1} E\left[Q_{l}(n)\right] E\left[\sin \left(\frac{2 \pi k n}{N}\right)\right]=0,
\end{aligned}
$$

where $E$ represents the expectation or the mean value. The real and imaginary parts of the complex quantization noise in the reconstruction plane thus have zero mean.

The variance of $R_{l}$ can be derived to be

$$
\begin{aligned}
\sigma_{R_{l}}^{2}= & E\left[R_{l}^{2}\right]=\frac{1}{N} \sum_{n=0}^{N-1} \sum_{m=0}^{N-1} E\left[Q_{l}(n) Q_{l}(m)\right] \\
& \times E\left[\cos \left(\frac{2 \pi k n}{N}\right) \cos \left(\frac{2 \pi k m}{N}\right)\right]
\end{aligned}
$$

For $\quad n \neq m, \quad E\left[\cos \left(\frac{2 \pi k n}{N}\right) \cos \left(\frac{2 \pi k m}{N}\right)\right]=E\left[\cos \left(\frac{2 \pi k n}{N}\right)\right]$ $E\left[\cos \left(\frac{2 \pi k m}{N}\right)\right]=0$, and only the $n=m$ terms contribute to the sum. Therefore,

$$
\begin{aligned}
\sigma_{R_{l}}^{2} & =\sum_{n=0}^{N-1} \frac{1}{N} E\left[Q_{l}(n)^{2}\right] E\left[\cos ^{2} \phi_{n}\right] \\
& =\frac{1}{N} \sum_{n=0}^{N-1} E\left[Q_{l}(n)^{2}\right] E\left[\frac{1}{2}+\frac{1}{2} \cos ^{2} \phi_{n}\right] \\
& =\frac{1}{N} \sum_{n=0}^{N-1} \frac{E\left[Q_{l}(n)^{2}\right]}{2}=\frac{\sigma_{Q_{l}}^{2}}{2} .
\end{aligned}
$$

Similarly $\sigma_{I_{l}}^{2}=\frac{\sigma_{Q_{l}}^{2}}{2}$ where $\sigma_{Q}^{2}$ is the variance of $Q_{l}$. The statistical distributions of the amplitude and the intensity of the complex noise can also be derived. A complete derivation of these statistical distributions with the necessary assumptions and conditions can be found in [36-38]. These distributions are summarized in Table 1 for the case of a hologram having a quantization interval of $\Delta_{l}$, defined in Eq. (4).

The amplitude of $q_{l}$, denoted by $A_{l}$, is Rayleigh distributed and the intensity $\mathrm{Int}_{l}$ is exponentially distributed. We validate this theoretical model by calculating a requantized digital holograms with 4 bits, $H_{4}$ from $H_{12}$ by uniformly quantizing. We can reconstruct these two digital holograms, $H_{4}$ and $H_{12}$, using a FFT to obtain the complex reconstructions $h_{12}$ and $h_{4}$. In Eq. (5), we showed that $h_{l}=h+q_{l}$ where $h$ was an ideally reconstructed digital hologram. We can write $q_{l}$ as the difference between the reconstruction of the quantized hologram, $h_{l}$, and the noise free reconstruction, $h$. If we assume that for the 12 bit case, $Q_{12} \approx 0$ and $H_{12} \approx H$, we can further assume that $h_{12} \approx h$ and $q_{12} \approx 0$. Therefore, we can calculate $q_{l}$ using the equation

$$
q_{l}=h_{12}-h_{l} .
$$

We can thus determine the error in the reconstruction for any quantization rate. In Fig. 9 , we show the theoretical and experimental histograms of the various parts of the complex noise for the quantization rate of 4 bits. As it can be seen in Fig. 9 , the statistics of quantization noise match the theoretically predicted values. We have found similar agreement between the theoretical model and the experimental results for quantization at other rates $(l=4$ bits to 11 bits).

In the previous sections, we examined the characteristics both of quantization noise in the hologram plane and the resulting noise in the reconstruction plane. The reconstruction, however, is the complex sum of both the complex reconstruction and the complex quantization noise. In the sections that follow, we look at the properties of this complex sum.

\section{Effect of Quantization Noise on Reconstructed Phase for Phase Objects}

The phase of the reconstructed image is important in digital holographic microscopy. For quantitative phase-contrast microscopy of transparent samples, information about the three dimensional structure is encoded in the phase variations of the reconstructed image. Skydan et al. [39] have studied the influence of quantization on the phase error in fringe

Table 1. Probability Densities of Different Parts of Quantization Noise in the Fourier Domain

\begin{tabular}{lc}
\hline \multicolumn{1}{c}{ Real Part $\left(R_{l}\right)$} & $p\left(R_{l}\right)=\frac{12}{\pi \Delta_{l}^{2}} \exp \left(\frac{-12 \mathrm{Re}_{l}^{2}}{\Delta_{l}^{2}}\right)$ \\
\hline Imaginary Part $\left(I_{l}\right)$ & $p\left(I_{l}\right)=\frac{12}{\pi \Delta_{l}^{2}} \exp \left(\frac{-12 \mathrm{Im}_{l}^{2}}{\Delta_{l}^{2}}\right)$ \\
Amplitude $\left(A_{l}\right)$ & $p\left(A_{l}\right)=\frac{24 A_{l}}{\Delta_{l}^{2}} \exp \left(\frac{-12 A_{l}^{2}}{\Delta_{l}^{2}}\right)$ \\
Phase $\left(\theta_{l}\right)$ & $p\left(\theta_{l}\right)=\frac{1}{2 \pi}$ \\
Intensity $\left(\operatorname{Int}_{l}\right)$ & $p\left(\operatorname{Int}_{l}\right)=\frac{12}{\Delta_{l}^{2}} \exp \left(\frac{-\operatorname{Int}_{l}}{\Delta^{2}}\right)$ \\
\hline
\end{tabular}




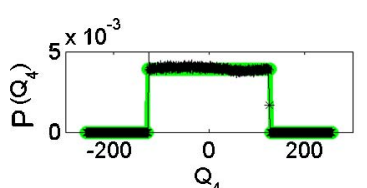

(a)

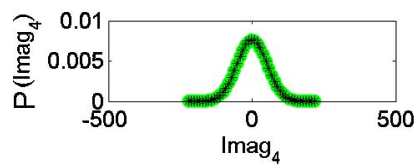

(c)

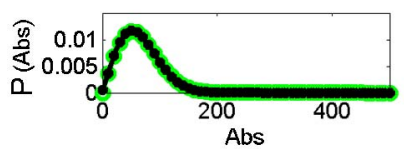

(e)

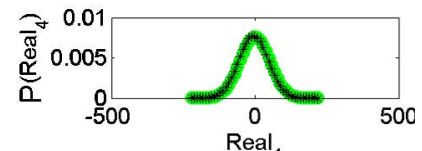

(b)

(d)

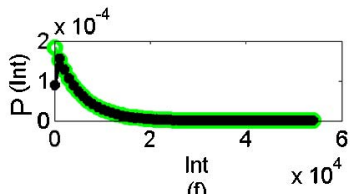

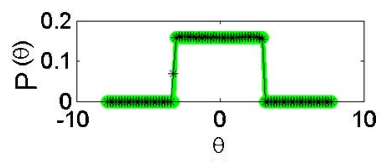

Fig. 9. (Color online) Theoretical and experimental probability distributions for various parts of the complex quantization noise in the Fourier plane for 4 bit quantization. (a) histogram of quantization noise in hologram plane. (b) distribution of real part of complex noise. (c) distribution of imaginary part of complex noise. (d) distribution of phase (e) distribution of amplitude. (f) distribution of intensity.

pattern analysis. In fringe pattern analysis, the phase measurement is performed on the recording plane whereas in digital holography, the phase measurement is performed on a Fourier or Fresnel transform plane. Digital holographic methods have been shown to give subwavelength axial resolution of a samples thickness and this is dependent on the accuracy of the phase measurement [6].

The value at every point in our reconstructed image is the complex sum of the ideal reconstruction, $h$ and the complex quantization noise, $q_{l}$. The addition of these two vectors is illustrated in Fig. 10. The resulting phase of the two vectors is different from the original phase. As seen in the Table 1, the phase of the noise in the Fourier domain, $\theta_{l}$ is uniformly distributed between $-\pi$ and $\pi$ and the amplitude of the noise, $A m_{l}$ is Rayleigh distributed. As seen in Fig. 10, the maximum error in the phase occurs when the noise vector is perpendicular to the signal vector, and the minimum, when the noise vector is parallel to the signal vector. It is of considerable interest to evaluate the influence of quantization error in phase angle measurement in a statistical manner. We assume that the amplitude of the transmission function of the object under investigation is uniform. For pure phase objects, this is a reasonably good assumption. We denote this amplitude as $A$. Since we measure the error in phase by subtracting the original phase distribution from the erroneous one, this problem is completely analogous to the phase distribution in the resulting sum of a speckle pattern and a coherent background [38], and in the case of coherent noise in MRI data [40]. In the case of speckle on a coherent background, the background is coherent and uniform, the speckle adds to this coherent background, and the resulting phase is given by the distribution above.

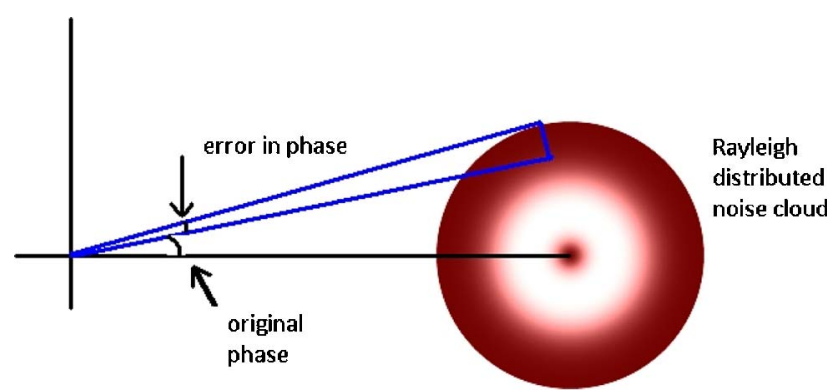

Fig. 10. (Color online) Complex quantization noise, $q_{l}$, is distributed with a Rayleigh distribution and adds on to the original amplitude vector. The net phase detected is the angle subtended by the complex vector sum of the original signal and the complex noise vector.

The phase error can be defined as the difference between the phase of the original signal $\left(S_{o}\right)$ and the new noisy signal $\left(S_{n}\right)$. The phase error can then be described as

$$
\theta_{\text {err }}=\angle S_{n}-\angle S_{o} .
$$

If $S_{o}=A \exp \left(i \angle S_{o}\right)$, then $S_{n}=S_{o}+N$, and $\theta_{\text {err }}$ can be written as

$$
\begin{aligned}
\theta_{\text {err }} & =\angle S_{n}-\angle S_{o}=\angle\left[S_{n} \exp \left(-i \angle S_{o}\right)\right] \\
& =\angle\left[A+N \exp \left(-i \angle S_{o}\right)\right] .
\end{aligned}
$$

In our case, the background is the noise-free reconstruction of the phase and is not uniform. But since we calculate the phase error by subtracting the noise-free phase reconstruction from the noisy one, our situation is similar to the one of the speckle against a coherent background.

The probability density for such a phase distribution can be derived to be [1]

$$
\begin{aligned}
p(\theta)= & \frac{1}{2 \pi} \exp \left(\frac{-A^{2}}{2 \sigma_{R}^{2}}\right)\left\{1+\frac{A}{\sigma_{R}} \sqrt{2 \pi} \cos \theta\right. \\
& \left.\times \exp \left(\frac{A^{2} \cos ^{2} \theta}{2 \sigma_{R}^{2}}\right)\left[1-\Phi\left(\frac{A \cos \theta}{\sigma_{R}}\right)\right]\right\},
\end{aligned}
$$

where

$$
\Phi(x)=\frac{1}{2 \pi} \int_{x}^{\infty} \exp \left(-x^{2} / 2\right) \mathrm{d} x,
$$

and $\sigma_{R}$ is the standard deviation in the real part, $R$. The probability density function is symmetric around the origin as the error can fluctuate in both the directions. For $A=0$, the phase is distributed uniformly between $-\pi$ to $\pi$. For high values of $A$, the error becomes Gaussian and centered around 0 with standard deviation of $\sigma_{R} / A$ and Eq. (17) density can be approximated as 


$$
p\left(\theta_{\mathrm{err}}\right)=\frac{A}{\sqrt{2 \pi} \sigma_{R}} \exp \left(\frac{-\theta_{\mathrm{err}}^{2} A^{2}}{2 \sigma_{R}^{2}}\right) .
$$

This can be expected as the magnitude of the corrupting phasors becomes small relative to the original signal magnitude. For a high number of bits per pixel representation, the error will be small. From Eq. (19), the standard deviation in the error in terms of the quantization interval can be written as

$$
\sigma_{\theta_{\mathrm{err}}}=\frac{M_{x}-M_{n}}{2^{l} \sqrt{24} A}=\frac{\sigma_{Q_{l}}}{\sqrt{2} A},
$$

where $M_{x}$ and $M_{n}$ as defined previously are the maximum and minimum values in the recorded digital hologram and $A$ is the average amplitude value in the reconstructed object area. $A$ is directly proportional to the product of object and reference powers $(O R)$ in the hologram plane.

We experimentally verify Eq. (22) by recording a hologram of a lens using the same experimental setup as shown in Section 2. This time the illumination is without a diffuser. The object beam passes through a aperture of a small lens. The recorded off-axis hologram and the reconstructed phase are shown in Fig. 11. Figure 11(a) shows the recorded phase hologram. Figure 11(b) shows the reconstructed image. The spatially separated areas carrying the object and the twin image can be clearly seen. Figure 11(c) shows the amplitude in a section of that area and Fig. 11(d) shows the phase value in that section. We are interested in the error which occurs in this phase section when the hologram is quantized to lower number of levels. We requantize the recorded hologram and the phase of the new noisy reconstruction is calculated and subtracted from the original phase of $h_{12}$ to give the phase error. The phase error varies between $(-\pi$ to $+\pi)$. The distribution of the phase error for four different cases: (a) 8 bits, (b) 6 bits, (c) 4 bits, and (d) 1 bit is shown in Fig. 12. As can be seen, the phase error can be modeled as a Gaussian density function for all these cases.

In our case, the mean amplitude value in the object area in the reconstruction, $A$ is 953 , and the fluctuation around this value due to the various experimental issues such as spurious reflections, etc., is less than $20 \%$. This value is directly proportional to the mean amplitude in the reconstructed object area. In order to compare the theoretical probability $p\left(\theta_{\text {err }}\right)$ in Eq. (19) with the histogram obtained from the experimental data, the theoretical value of standard deviation in the phase error is plotted with the experimentally obtained value for different levels of quantization in Fig. 13. The curves show a close agreement. The standard deviation in the phase error decreases by a factor of 2 for every bit added. For 8 bits of quantization, the standard deviation in the error is $\sigma_{\theta \text { orr }}=0.00247 \mathrm{rad}$. This corresponds to approximately $0.0004 \lambda$ error in surface height measurement in air for a reflection geometry. For the 1 bit case, the $\sigma_{\theta_{\text {err }}}=0.3165 \mathrm{rad}$ and this corresponds to

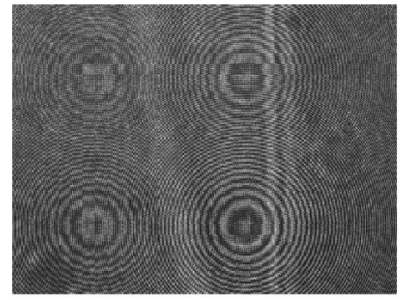

(a) Hologram

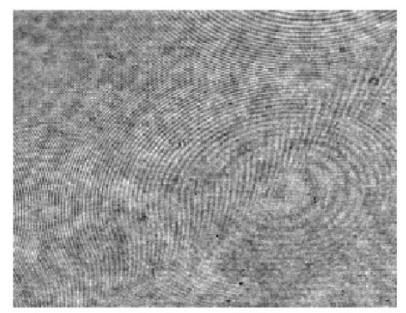

(c) Amplitude(reconstruction)

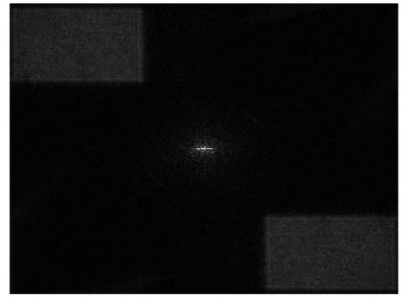

(b) Reconstruction

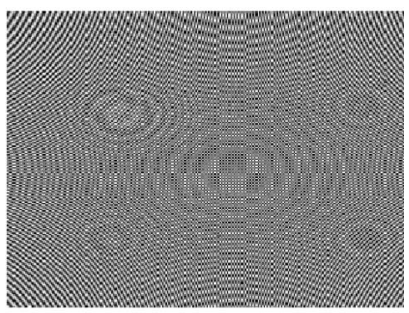

(d) Phase(reconstruction)
Fig. 11. (a) Recorded phase hologram of the a lens, (b) Fourier reconstruction, (c) amplitude in a $200 \times 250$ section of the reconstructed object, (d) phase distribution in the same area as (c).

approximately $0.05 \lambda$ error in surface height measurement in air.

As seen in Eq. (19), the error in the phase resulting from quantization can be reduced by having a large mean amplitude in the reconstruction relative to the standard deviation in the error introduced due to the quantizer. The mean amplitude can be easily shifted by increasing the exposure. Thus it is advantageous to have a high mean intensity in the reconstruction to reduce the influence of quantization error on the phase measurement.

\section{Effect of Quantization Noise on Reconstructed Intensity for 3D Objects}

Many applications of digital holography such as 3D display, pattern recognition, imaging through occlusions [42], and projection on 2D surfaces [43] rely on the intensity pattern in the reconstruction plane. In this subsection, we analyze the statistics in the

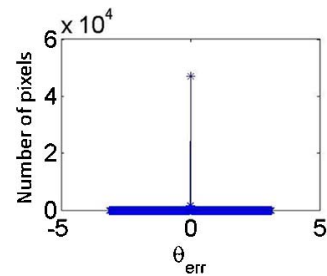

(a) 8 bits

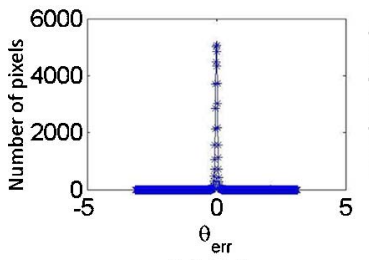

(c) 4 bits

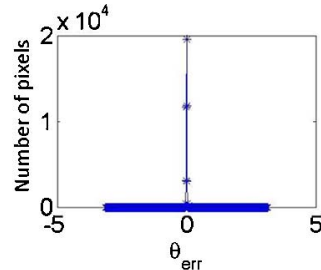

(b) 6 bits

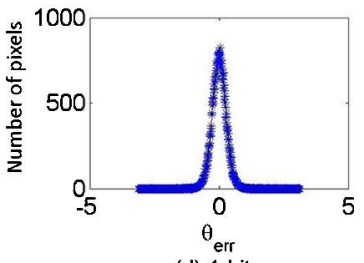

(d) 1 bits
Fig. 12. (Color online) Error distribution in phase $-\pi$ to $+\pi$ for (a) 8 bits, (b) 6 bits, (c) 4 bits, (d) 1 bit. 
reconstructed intensity when quantization noise $q_{l}$ is present. The intensity of the hologram is

$$
\left|h_{l}\right|^{2}=\left|h+q_{l}\right|^{2}=|h|^{2}+\left|q_{l}\right|^{2}+h q_{l}^{*}+h^{*} q_{l},
$$

which can be written as

$$
\begin{aligned}
\left|h_{l}\right|^{2}= & |h|^{2}+\left|q_{l}\right|^{2} \\
& +2\left(\operatorname{Real}[h] \operatorname{Real}\left[q_{l}\right]-\operatorname{Imag}[h] \operatorname{Imag}\left[q_{l}\right]\right) .
\end{aligned}
$$

This is the sum of four terms, all of which are independent of each other. Since $h$ and $q_{l}$ are independent and the real part and imaginary parts are uncorrelated with each other [38], the variance in the sum is equal to the sum of the variances. The total variance in the complex intensity in the Fourier plane can be approximately written as

$$
\begin{aligned}
\sigma_{\left|h_{l}\right|^{2}}^{2}= & \sigma_{|h|^{2}}^{2}+\sigma_{\left|q_{l}\right|^{2}}^{2}+4 \sigma_{\operatorname{Real}(|h|)}^{2} \sigma_{\operatorname{Imag}\left(\left|q_{l}\right|\right)}^{2} \\
& +4 \sigma_{\operatorname{Imag}(|h|)}^{2} \sigma_{\operatorname{Real}\left(\left|q_{l}\right|\right)}^{2}
\end{aligned}
$$

Since $\sigma_{\text {Real }(|h|)}^{2}$ and $\sigma_{\operatorname{Imag}(|h|)}^{2}$ are known a priori, we can use this simple model to calculate the standard deviation in the image for any lower number of quantization levels. $\sigma_{\text {Real }(|h|)}^{2}$ and $\sigma_{\operatorname{Imag}(|h|)}^{2}$ are assumed in our case to be equal to $\sigma_{\operatorname{Real}\left(\left|h_{12}\right|\right)}^{2}$ and $\sigma_{\operatorname{Imag}\left(\left|h_{12}\right|\right)}^{2}$, respectively. In Fig. 14, we plot the standard deviation in a uniform region in the reconstructed image along with the value calculated using Eq. (23). The observed value matches the predicted value with high accuracy down to 2 bits. For 1 bit quantization, the predicted value deviates from the observed value. At 1 bit, the deviation is $26 \%$. We believe that is due to the onset of "banding" as explained previously. In Section 4, we discuss a method to suppress the noise cause $\bar{d}$ by quantization in the reconstructed intensity for the particular application of 3D display.

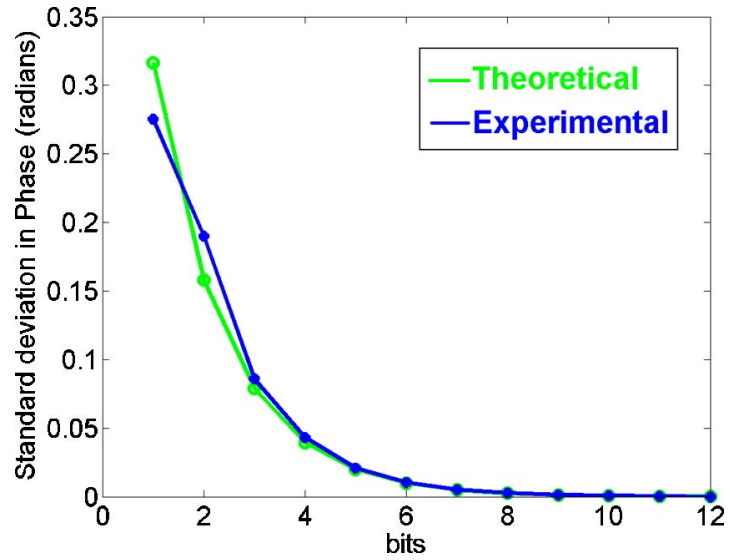

Fig. 13. (Color online) Standard deviation of the error in reconstructed phase, $\theta_{\text {err }}$ for different levels of quantization of the recorded phase hologram.

\section{Suppression of Quantization Noise}

Speckle is present in digital holographic imaging of objects with rough surfaces or diffusely illuminated objects. The image quality of objects containing speckle can be considerably improved by performing speckle reduction $[44,45]$. In Section 3 , we showed that since the quantization noise occurs in the hologram plane as a white noise, its DFT can be treated like a random walk and can be assumed to be a 'speckle like' distribution. Thus it can be viewed as an additional "speckle-like" noise occurring in the reconstruction plane. This leads to the possibility of suppressing the noise in the reconstruction plane by using a simple speckle reduction technique of adding independent speckle patterned reconstructions [46].

This is achieved by moving a diffuser to generate statistically independent speckle patterns. Since the speckle pattern is generated by a moving diffuser with approximately uniform transmittance and the quantization rate is constant for all the different captures, the mean value of these intensity patterns is approximately constant. When $N$ statistically independent intensities are added together, the variance of the sum increases $N$ times and the standard deviation increases $\sqrt{N}$ times but the mean value of the sum increases $N$ times. Thus the coefficient of variation, also called the speckle index [47-49], which is the ratio of the standard deviation to the mean, in this case decreases as $1 / \sqrt{N}$.

We experimentally prove this by capturing a number of different statistically independent speckle patterns of the object. This is achieved by moving a diffuser on a translational stage with steps of $1 \mathrm{~mm}$. The resulting holograms are quantized to the binary level ( 1 bit per pixel) and reconstructed. The reconstructed intensities for all the different reconstructions are added together. Speckle reduction

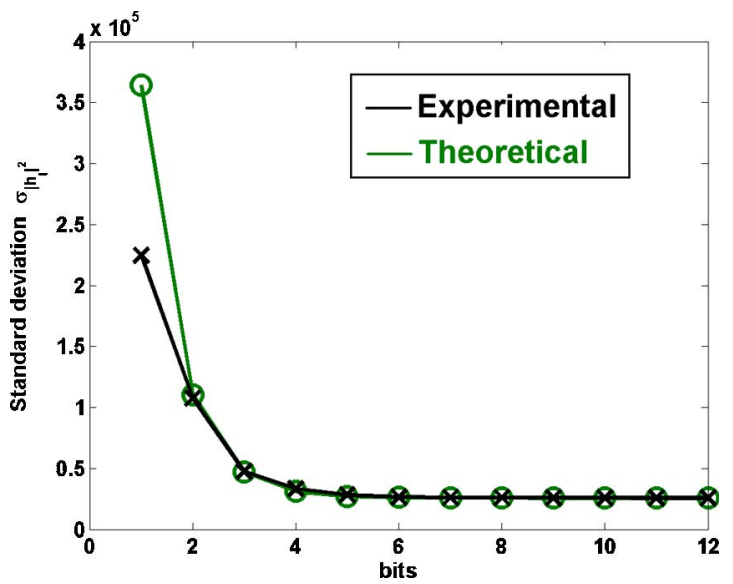

Fig. 14. (Color online) Standard deviation in a uniform section of the intensity of the reconstruction plane. We can see that the simple model above can be used to predict accurately the quantization error upto 2 bits ( 4 levels) of quantization. The deviation at 1 bit is due to the nonwhite nature of quantization noise in the hologram plane at binary quantization. In this case, the noise term has a pattern and structure similar to the original signal. 
dramatically increases the quality of reconstruction. In Fig. 15, the speckle index is plotted with respect to the number of diffusers added. The results show a well matched $1 / \sqrt{N}$ dependence. The results are shown for the extreme 1 bit case where the quantization noise is at a maximum. We mention that in the case of Fresnel geometry, where the twin image occurs as an out of focus noise, speckle reduction can be used to suppress the twin image [50].

A single hologram acquired by a typical camera requires 12 bits per pixel which uses the same memory as that of twelve 1 bit holograms. To give another comparison with respect to memory usage, we show in Fig. 16 two reconstructions, one from an individual 12 bit hologram and the other the intensity summed from twelve 1 bit holograms. The summation from twelve 1 bit holograms gives a better reconstruction than the case from one 12 bit hologram, even though both of them utilize the same memory. Of course, it can be argued that this method of capturing requires more time and requires moving diffusers but there are significant advantages like better contrast and improved detail in the reconstruction for the same memory of storage of holograms. Since a binary capture sequence is inherently fast on hardware, the capture time can be reduced to be the same as that of a single 12 bit or 8 bit image capture. Another advantage is that binary ferroelectric SLMs have much faster switching rates (in $\mathrm{KHz}$ ) than multiphase LCOS SLMs, which are around 80-90 frames/s. Thus multiple binary holograms could be captured, transmitted over network/hardware bus with minimal latency and displayed in a fast amplitude or phase SLM for 3D display and projection applications. When a captured hologram is displayed on a spatial light modulator, this can be used to generate improved reconstructions. The different speckled holograms are displayed at a fast frame rate, such that many holograms are averaged over the integration

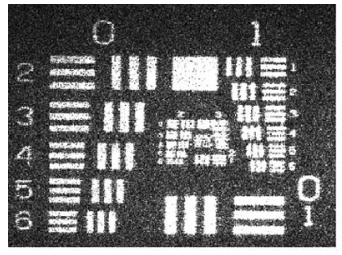

(a)

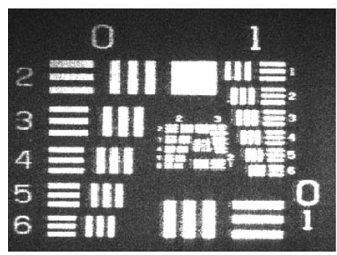

(c)

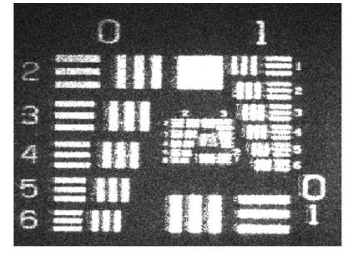

(b)

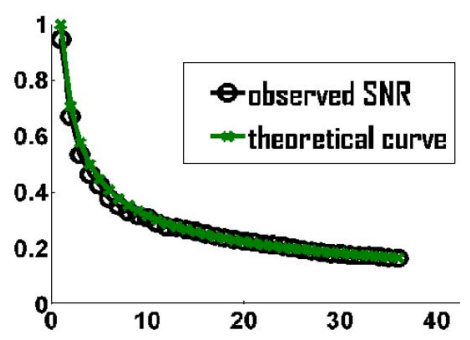

(d)
Fig. 15. (Color online) Results for speckle reduction on the 1 bit holograms (a) four diffuse holograms added together, (b) 16 patterns, (c) 36 patterns, (d) speckle index in the image.

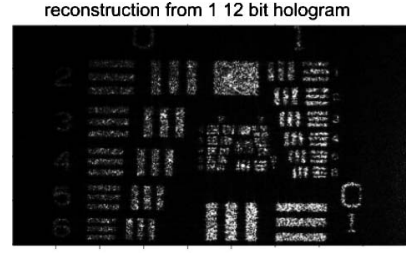

(a)

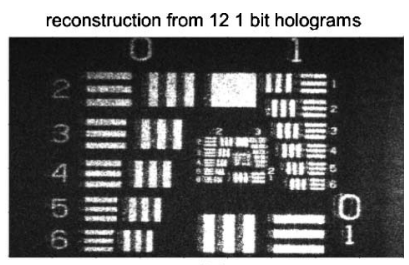

(b)
Fig. 16. (Color online) Comparison of reconstructions from (a) a single 12 bit hologram and (b) reconstructions obtained from 12 1 bit holograms. The two reconstructions involve the same amount of memory usage (in bits).

time of the eye and a speckle and quantization noise reduced image is perceived. This technique has been demonstrated in the area of computer generated holographic projection where the different holograms are computed and given a random phase pattern [43]. We have obtained the same results for reflective objects as well.

\section{Applications to Noise from Other Sources}

Quantization noise is only one of many noise sources in digital holographic imaging. Other important sources include:

- Shot noise, which is predominant in low-light level imaging. This noise follows Poisson statistics and is inherently present due to the random nature of the photon emission from the laser source. At high light intensities, this noise is negligible, but it is the ultimate noise limit in imaging. Charriere et al. [51] have studied the influence of this noise in detail in holographic microscopy and Gross et al. [52] have showed a technique for holographic imaging at low intensities.

- Thermal noise in camera photodetectors, resulting from random thermal fluctuations in the photodetectors.

- Pixel nonuniformity noise (PRNU), from slight variation in response from each pixel [53] and pixel crosstalk, between neighboring pixels.

- Computational noise due to the hardware implementation and finite bit representation of the numerical algorithms. This noise is extremely low for modern 64 bit precision computers but is still a noise source and can depend on the algorithm used for reconstructing the image.

- Noise due to vibrations, dust particles, coherent reflections, etc.

- Additionally, transmission of digital holograms on a channel without error correction can lead to "channel noise," where the bits can be lost or assigned a different value.

These noises can be modeled as additive noises with their own statistical distributions and the statistics in the Fourier domain can be derived. Therefore, we believe that the analysis in this paper can be extended to any additive noise in the hologram plane and the influence of all these noise sources can be 
reduced by adding multiple differently captured images.

\section{Conclusion}

Quantization error is fundamental to any digital sensor. We have shown that in digital holography, quantization error is introduced as uniformly distributed additive noise in the recording plane (camera plane) and this manifests itself as a complex noise in the reconstruction plane with Gaussian distributed real and imaginary parts, rayleigh distributed amplitude and uniformly distributed phase. We have shown and characterized the various statistical properties of this noise both theoretically and experimentally in the case of lensless off-axis Fourier digital holography. We have also discussed the nonwhite nature of quantization noise at low bit rate (of binary quantization) in digital holography for the first time. We have studied the influence on the phase measurement accuracy and the reconstructed intensity quantitatively. The standard deviation in the phase measurement error increases by a factor of 2 for every bit removed in the quantization process. The effect of quantization noise on the phase can be suppressed (linearly) by increasing the mean intensity of the phase hologram during recording. We have also characterized the noise in the intensity of the reconstructed images. This complex noise can be treated as the result of a random walk in the complex plane and shares the same statistical properties as that of speckle. We have suppressed this noise by using a speckle reduction technique and have shown good quality reconstructions with binary holograms when speckle reconstruction is performed. We have shown improved reconstructions while using the same amount of memory in storing the holograms. Though we have studied the noise due to quantization, we believe that the same method can be applied to other kinds of additive noise in the hologram plane.

The research leading to these results has received funding from the European Community's Seventh Framework Programme FP7/2007-2013 under grant 216105, and from Science Foundation Ireland under the National Development Plan. All of the experimental data, digital holograms, and computer code used in this analysis can be found at http://www.cs .nuim.ie/ npandey.

\section{References}

1. J. Goodman and R. Lawrence, "Digital image formation from electronically detected holograms," Appl. Phys. Lett. 11, 77 (1967).

2. M. Kronrod, N. Merzlyakov, and L. Yaroslavskii, "Reconstruction of a hologram with a computer," in SPIE Milestone Series 144 (SPIE Press, 1998), pp. 645-646.

3. L. Yaroslavskii and N. Merzlyakov, Methods of Digital Holography (Consultants Bureau, 1980).

4. U. Schnars and W. Jüptner, "Direct recording of holograms by a CCD target and numerical reconstruction," Appl. Opt. 33, 179-181 (1994).

5. T. Colomb, J. Kühn, F. Charriere, C. Depeursinge, P. Marquet, and N. Aspert, "Total aberrations compensation in digital ho- lographic microscopy with a reference conjugated hologram," Opt. Express 14, 4300-4306 (2006).

6. P. Marquet, B. Rappaz, P. Magistretti, E. Cuche, Y. Emery, T. Colomb, and C. Depeursinge, "Digital holographic microscopy: a noninvasive contrast imaging technique allowing quantitative visualization of living cells with subwavelength axial accuracy," Opt. Lett. 30, 468-470 (2005).

7. T. Baumbach, W. Osten, C. von Kopylow, and W. Jüptner, "Remote metrology by comparative digital holography," Appl. Opt. 45, 925-934 (2006).

8. T. Poon, Digital Holography and Three-Dimensional Display: Principles and Applications (Springer, 2006).

9. N. Pandey and B. Hennelly, "Fixed-point numercial-reconstruction for digital holographic microscopy," Opt. Lett. 35, 1076-1078 (2010).

10. T. Naughton, Y. Frauel, B. Javidi, and E. Tajahuerce, "Compression of digital holograms for three-dimensional object reconstruction and recognition," Appl. Opt. 41, 4124-4132 (2002).

11. T. Naughton, J. McDonald, and B. Javidi, "Efficient compression of Fresnel fields for internet transmission of threedimensional images," Appl. Opt. 42, 4758-4764 (2003).

12. T. Naughton and B. Javidi, "Compression of encrypted threedimensional objects using digital holography," Opt. Eng. 43, 2233 (2004).

13. O. Matoba, T. Naughton, Y. Frauel, N. Bertaux, and B. Javidi, "Real-time three-dimensional object reconstruction by use of a phase-encoded digital hologram," Appl. Opt. 41, 6187-6192 (2002).

14. A. Shortt, T. Naughton, and B. Javidi, "A companding approach for nonuniform quantization of digital holograms of three-dimensional objects," Opt. Express 14, 5129-5134 (2006).

15. A. Shortt, T. Naughton, and B. Javidi, "Compression of optically encrypted digital holograms using artificial neural networks," J. Display Technology 2, 401-410 (2006).

16. A. Shortt, T. J. Naughton, and B. Javidi, "Compression of digital holograms of three-dimensional objects using wavelets," Opt. Express 14, 2625-2630 (2006).

17. G. Mills and I. Yamaguchi, "Effects of quantization in phase-shifting digital holography," Appl. Opt. 44, 1216-1225 (2005).

18. E. Darakis and J. Soraghan, "Reconstruction domain compression of phase-shifting digital holograms," Appl. Opt. 46, 351-356 (2007).

19. E. Darakis, T. Naughton, and J. Soraghan, "Compression defects in different reconstructions from phase-shifting digital holographic data," Appl. Opt. 46, 4579-4586 (2007).

20. A. Gotchev and L. Onural, "A survey on sampling and quantization in diffraction and holography," in Workshop on Spectral Methods and Multirate Signal Processing, SMMSP (2006), pp. 179-190.

21. D. Psaltis, E. Paek, and S. Venkatesh, "Optical image correlation with a binary spatial light modulator," in SPIE Milestone Series, 156 (SPIE Press, 1999), pp. 482-488.

22. B. Javidi and J. Horner, "Single spatial light modulator joint transform correlator," Appl. Opt. 28, 1027-1032 (1989).

23. A. Bourquard, F. Aguet, and M. Unser, "Optical imaging using binary sensors," Opt. Express 18, 4876-4888 (2010).

24. W. Dallas and A. Lohmann, "Phase quantization in holograms-depth effects," Appl. Opt. 11, 192-194 (1972).

25. A. W. Lohmann and D. P. Paris, "Binary Fraunhofer holograms, generated by computer," Appl. Opt. 6, 1739-1748 (1967).

26. F. Wyrowski, "Iterative quantization of digital amplitude holograms,” Appl. Opt. 28, 3864-3870 (1989). 
27. R. Powers and J. Goodman, "Error rates in computergenerated holographic memories," Appl. Opt. 14, 1690-1701 (1975).

28. P. Naidu, "Quantization noise in binary holograms," Opt. Commun. 15, 361-365 (1975).

29. M. Seldowitz, J. Allebach, and D. Sweeney, "Synthesis of digital holograms by direct binary search," Appl. Opt. 26, 2788-2798 (1987).

30. L. Schuchman, "Dither signals and their effect on quantization noise," IEEE Trans. Communication Technology 12, 162-165 (1964).

31. J. Goodman, Introduction to Fourier Optics (Roberts \& Co., 2005).

32. D. Kelly, B. Hennelly, N. Pandey, T. Naughton, W. Rhodes, "Resolution limits in practical digital holographic systems," Opt. Eng. 48, 095801 (2009).

33. H. Jin, H. Wan, Y. Zhang, Y. Li, and P. Qiu, "The influence of structural parameters of CCD on the reconstruction image of digital holograms," J. Mod. Opt. 55, 2989-3000 (2008).

34. A. Oppenheim and R. Schafer, Discrete-Time Signal Processing (Prentice-Hall, 1999).

35. A. Sripad and D. Snyder, "A necessary and sufficient condition for quantization errors to be uniform and white," IEEE Trans. Acoust. Speech Signal Process. 25, 442-448 (1977).

36. J. Schoukens and J. Renneboog, "Modeling the noise influence on the Fourier coefficients after a discrete Fourier transform," IEEE Trans. Instrum. Meas. 35, 278-286 (1986).

37. R. Shiavi, Introduction to Applied Statistical Signal Analysis: Guide to Biomedical and Electrical Engineering Applications (Academic, 2007).

38. J. Goodman, Speckle Phenomena in Optics: Theory and Applications (Roberts \& Co, 2007).

39. O. A. Skydan, F. Lilley, M. J. Lalor, and D. R. Burton, "Quantization error of ccd cameras and their influence on phase calculation in fringe pattern analysis," Appl. Opt. 42, 5302-5307 (2003).
40. H. Gudbjartsson and S. Patz, "The Rician distribution of noisy MRI data," Magnetic Resonance Medicine 34, 910-914 (1995).

41. D. Middleton, An Introduction to Statistical Communication Theory (McGraw-Hill, 1960).

42. J. Maycock, C. Elhinney, B. Hennelly, T. Naughton, J. McDonald, and B. Javidi, "Three-dimensional scene reconstruction of partially occluded objects using digital holograms," Appl. Opt. 45, 2975-2985 (2006).

43. E. Buckley, "Holographic laser projection technology," Information Display 24, 12 (2008).

44. P. Hariharan and Z. Hegedus, "Reduction of speckle in coherent imaging by spatial frequency sampling," J. Mod. Opt. 21, 345-356 (1974).

45. J. Dainty, "Laser speckle and related phenomena," in Topics in Applied Physics (Springer-Verlag, 1975), Vol. 9, p. 298.

46. J. W. Goodman, "Some fundamental properties of speckle," J. Opt. Soc. Am. 66, 1145-1150 (1976).

47. J. Maycock, B. Hennelly, J. McDonald, Y. Frauel, A. Castro, B. Javidi, and T. Naughton, "Reduction of speckle in digital holography by discrete Fourier filtering," J. Opt. Soc. Am. 24, 1617-1622 (2007).

48. J. Lim and H. Nawab, "Techniques for speckle noise removal," Opt. Eng. 20, 472-480 (1981).

49. T. Crimmins, "Geometric filter for speckle reduction," Appl. Opt. 24, 1438-1443 (1985).

50. D. Monaghan, D. Kelly, N. Pandey, and B. Hennelly, "Twin removal in digital holography using diffuse illumination," Opt. Lett. 34, 3610-3612 (2009).

51. F. Charriére, B. Rappaz, J. Kühn, T. Colomb, P. Marquet, and C. Depeursinge, "Influence of shot noise on phase measurement accuracy in digital holographic microscopy," Opt. Express 15, 8818-8831 (2007).

52. M. Gross and M. Atlan, "Digital holography with ultimate sensitivity," Opt. Lett. 32, 909-911 (2007).

53. J. Lukas, J. Fridrich, and M. Goljan, "Digital camera identification from sensor pattern noise," IEEE Trans. Info. Foren. Sec. 1, 205-214 (2006). 